\title{
Tick-Borne Relapsing Fever
}

National Cancer Institute

\section{Source}

National Cancer Institute. Tick-Borne Relapsing Fever. NCI Thesaurus. Code C34976.

An infection that is caused by certain species of Rickettsia or Borrelia, which are transmitted to humans from infected ticks; it is characterized by sudden fever, chills, headaches, myalgia, arthralgia, nausea, and possibly a rash. Symptoms usually persist for two to nine days, then disappear, with recurrence after several weeks if the patient remains untreated. 